\title{
Caregiving for Older Adults with Limited English Proficiency: Transitioning from Hospital to Home
}

\author{
Wagahta Semere, $M D, M H S^{1,2}$, Anna María Nápoles, $\mathrm{PhD}, \mathrm{MPH}^{3}$, \\ Steven Gregorich, $P h D^{1,4}$, Jennifer Livaudais-Toman, $P h D^{1,4}$, and Leah Karliner, $M D, M A S^{1,4,5}$

'Department of Medicine, University of California, San Francisco, CA, USA; ${ }^{2}$ Zuckerberg San Francisco General, San Francisco, CA, USA; ${ }^{3}$ National Institute on Minority Health and Health Disparities, National Institutes of Health, Bethesda, MD, USA; ${ }^{4}$ Multiethnic Health Equity Research Center, San Francisco, CA, USA; ${ }^{5}$ UCSF, San Francisco, CA, USA.

BACKGROUND: Although the family caregiver workforce is increasingly diverse, little is known about culturally and linguistically diverse caregivers and patients for whom they care. Caregiver roles include communicating with health care teams on behalf of patients with language barriers.

OBJECTIVE: Our objective is to describe characteristics and experiences of caregivers for patients with limited English proficiency (LEP) immediately following hospital discharge.

DESIGN: Cross-sectional.

PARTICIPANTS: Primary informal caregivers for Chineseand Spanish-speaking patients with LEP discharged from a large academic medical center's orthopedic surgery, general surgery, and cardiovascular inpatient floors from June 2012 to August 2013.

MAIN MEASURES: Bilingual-bicultural research assistants conducted baseline structured interviews with patients or surrogates in the hospital, and 3 weeks after discharge, gathering demographic and health information. They then interviewed by phone informal caregivers, identified by patients, to determine caregiving experiences.

KEY RESULTS: One hundred fifty-eight caregivers were interviewed post-discharge. Two-thirds (69.0\%) were adults caring for parents or grandparents, and $20.9 \%$ were spouses or partners. Sixty-nine (43.7\%) caregivers had LEP themselves, yet only $12 \%$ of patients reported having access to professional interpreters at the time discharge instructions were provided. Ninety percent reported performing three or more caregiving roles for the patient (helping at home, helping with medical decisions, helping with medical forms, helping communicate with medical staff, and talking with doctors about medical care). Forty percent reported moderate/high levels of perceived stress (some, most, or all of the time) caring for the patient. Multivariate regression revealed caregivers for Chinese-speaking patients, and those for patients discharged to another hospital were most likely to report moderate/high levels of perceived stress.

Prior Presentations We presented early findings from this manuscript as a poster at the Society of General Internal Medicine annual meeting in Toronto, Canada, in 2015.

Received May 14, 2018

Revised October 26, 2018

Accepted April 10, 2019

Published online June 24, 2019
CONCLUSION: Culturally and linguistically diverse caregivers perform multiple roles caring for patients with LEP, often have LEP themselves, and experience notable levels of stress. These results also demonstrate an opportunity to expand the use of professional interpreters at hospital discharge to avoid communication errors.

KEY WORDS: caregiving; care transitions; limited English proficiency; geriatrics.

J Gen Intern Med 34(9):1744-50

DOI: $10.1007 / \mathrm{s} 11606-019-05119-\mathrm{y}$

(c) Society of General Internal Medicine 2019

\section{INTRODUCTION}

Family (or informal) caregivers have a significant role in home care of elderly patients. In 2015, of the 43.5 million Americans who reported providing unpaid care during the past year, $79 \%$ cared for an adult age 50 or older. ${ }^{1}$ Beyond assisting with activities of daily living, caregivers are often involved in medical decisions, patient advocacy, and communication with health care providers; they also provide emotional and social support to patients. ${ }^{2,3}$ The volume of caregiver responsibility coupled with lack of formal support systems and training for these roles can lead to caregiver burden. A recent national survey found that 4 in 10 caregivers consider their caregiving situation to be highly emotionally stressful. ${ }^{1}$ These caregivers may suffer from decline in their own physical health, emotional well-being, and socioeconomic status. ${ }^{4-6}$ Caregivers responsible for patients with chronic conditions, limited mobility, medical or nursing needs, and extended care needs appear to experience the highest levels of emotional stress. $1,7,8$

Thus far, the majority of work examining caregivers and caregiver burden has focused on the outpatient setting, with limited data on experiences of culturally and linguistically diverse populations. Yet, over half of caregivers report that their care recipient was hospitalized at least once during the prior year and the caregiver workforce is increasingly diverse with Latinos accounting for nearly $20 \%$ of informal caregivers. ${ }^{1}$ A systematic review of ethnically diverse caregivers demonstrated numerous differences in caregiver experiences 
exist across cultural groups; however, few caregiver intervention studies report their findings stratified by cultural groups. ${ }^{9}$ An analysis of the American Time Use Survey (ATUS), reporting stratified results, found that Latino caregivers tend to engage in more time-intensive and demanding caregiving roles when compared with non-Latino Whites. Asian caregivers had more care recipients than other racial/ethnic groups. ${ }^{10}$ Little attention, however, has been given to describing in detail characteristics of culturally and linguistically diverse caregivers.

Patients with limited English proficiency (LEP) face challenges during hospitalization, including risk of errors and adverse events due to communication barriers. ${ }^{11}$ For caregivers with LEP, navigating the complexities of the health care system alongside patients with LEP compounds communication challenges and could lead to stress. The purpose of our study was to describe the demographic makeup, roles, and self-reported stress levels of caregivers for recently hospitalized Chinese- and Spanish-speaking patients with LEP, and to explore the characteristics of both caregivers and patients associated with elevated levels of caregiver perceived stress.

\section{METHODS}

\section{Study Design and Setting}

This was a cross-sectional study taking place at a large academic medical center with a diverse patient population. Approximately $24 \%$ of discharges per year involve patients with LEP. ${ }^{12}$ We investigated the characteristics and perspectives of caregivers for Chinese- and Spanish-speaking patients with LEP and their experiences during the period immediately following hospital discharge. The affiliated academic institutional review board approved this study.

\section{Study Population}

The study population was drawn from a parent study examining communication strategies among hospitalized patients with LEP. Details of this recruitment were previously published. ${ }^{13}$ In brief, we prospectively recruited Chinese- (Cantonese or Mandarin) and Spanish-speaking patients from orthopedic surgery, general surgery, and cardiovascular inpatient floors from June 2012 to August 2013. We interviewed patients in the hospital and asked them to identify their primary informal caregiver, who was present at the time of hospital discharge. Approximately 3 weeks after discharge, we then interviewed the caregiver by telephone.

Research assistants screened eligible patients to confirm patient age, LEP status using a validated LEP algorithm, ${ }^{14}$ and cognitive status using the Mini-Cog. ${ }^{15}$ Patients with cognitive impairment were included if they met all other inclusion criteria and a surrogate consented to participate in the study. Thirty-nine percent of patients had a surrogate, most of whom
$(90.3 \%)$ were also the identified primary caregiver. Patients were included in this analysis if they were alive at the time of post-discharge follow-up calls.

We conducted baseline structured interviews with patients or their surrogates in the hospital pre-discharge. Participants identified the primary caregiver, who was present at the time of hospital discharge. Caregiver was defined as "a relative, friend, or other caregiver present when you received instructions about your medicines, follow-up appointments, and how to care for yourself after leaving the hospital." We contacted identified caregivers for telephone interviews post-discharge; the mean time between patient discharge and caregiver interview was $39.7 \pm 15.8$ (SD) days. During the interview, each caregiver was screened to verify their role as the caregiver according to our study definition or to provide the name of another caregiver who met this criterion.

\section{Caregiver and Patient Characteristics}

Through the baseline interviews, we obtained demographics for patients including age, gender, country of birth, and highest level of education; we obtained the same information for caregivers at the follow-up caregiver interview. We additionally inquired about caregiver selfreported health. For patients, we asked about comorbidities, scored using the Self-Administered Comorbidity Questionnaire (SCQ), a validated instrument that uses simplified language to assess the presence of, treatment for, and limitation related to 12 common medical problems (score, range 0-36). ${ }^{16}$ We inquired about functional status, including activities of daily living (ADLs) and independent activities of daily living (IADLs). We abstracted discharge destination (home, SNF/rehab/hospice, another hospital) from patient charts. We inquired about both patient and caregiver health literacy, preferred language, and Englishspeaking ability using validated instruments. Limited English proficiency was defined using an algorithm validated in the clinical setting, which classifies patients as having LEP based on the US Census English proficiency definition and preferred language for receiving medical care. ${ }^{14}$ Health literacy was categorized as adequate or inadequate using a brief validated 1 question screening tool. ${ }^{17} \mathrm{We}$ asked all patients whether a professional interpreter was present at the time discharge medication and follow-up instructions were reviewed by the medical team.

During caregiver interviews, caregivers responded "yes" or "no" to assuming specific caregiving roles (helping at home, helping with medical decisions, talking to doctors about medical care, and helping communicate with the medical staff). We used a validated screening tool to assess caregiver global financial strain; specifically, we asked whether caregivers had difficulty paying monthly bills at any point over the past 12 months. ${ }^{18}$ Additionally, we used the Medical Outcomes Study of Social Support (MOS-SS) subscales of tangible and 
emotional/informational support (score, range 0-5) to assess caregivers' perceived support networks. ${ }^{19}$ For our primary outcome, we asked caregivers: "In the past week, how often have you felt stressed caring for the patient?" with response options "none of the time," "a little of the time," "some of the time," "most of the time," and "all of the time."

\section{Statistical Analysis}

Descriptive statistics were used to present demographic and health characteristics for patients and caregivers. We used Chisquare and Student's T tests to examine bivariate associations between caregiver self-reported stress level, and caregiver and patient characteristics. Caregiver stress levels were dichotomized as low stress ("none of the time" and "a little of the time") and moderate/high stress ("some of the time," "most of the time," and "all of the time"). Using a backward stepwise elimination approach, with a significance level of $p<0.20$, we selected variables to include in a multivariable logistic regression modeling the odds of reporting caregiver moderate/high stress versus low stress. Two-sided $p$ values $<0.05$ were considered statistically significant. We performed all statistical analyses using Stata 14 (StataCorp, LP, College Station, TX).

\section{RESULTS}

From the parent study recruitment, 254 patients were eligible to participate in the study and $84 \%(n=214)$ initially agreed to participation. The 39 patients that refused participation did not differ significantly from participants in terms of age, gender, or preferred language to receive medical care. In total, 202 (94.4\%) of 214 patients in the LEP cohort were alive at discharge. One hundred fifty-eight $(78.2 \%)$ caregivers for these patients completed follow-up interviews and were included in this analysis. Among the caregivers not included in the analysis, 10 refused participation, 21 were unable to be contacted despite three attempts, 12 cared for patients who died after discharge but before follow-up, and 2 did not respond to the caregiver perceived stress question.

\section{Caregiver Characteristics}

Two-thirds were adult children or grandchildren in a caregiver role. The median age of caregivers was 47 years (range 19-81) and a majority $(65.2 \%)$ were women. More than half reported living with the patient that they cared for. Overall, $41.7 \%$ of caregivers preferred to receive their own medical care in a language other than English, and 34.2\% reported speaking English not at all or not well. Twenty-nine percent of caregivers had inadequate health literacy (mean HL score $2.14 \pm$ 1.24). A quarter of caregivers did not have enough money to pay their monthly bills in the past year. Forty percent of caregivers felt stressed caring for their loved one at least some of the time (Table 1).
Caregivers reported multiple roles assisting patients post-hospitalization including helping at home (85.4\%), helping with medical decisions (79.6\%), helping with medical forms $(77.2 \%)$, talking with doctors $(88.6 \%)$, and communicating with medical staff $(84.6 \%)$. Ninety percent of caregivers reported taking on 3 or more caregiving roles for patients (Fig. 1).

\section{Patient Characteristics}

Overall, among the 158 patients cared for, the median age was 70 years (range 41-95) and a majority were women (58.2\%). Almost all patients preferred to receive medical care in a language other than English, and $83.8 \%$ had inadequate health literacy. Twelve percent of patients reported having a professional interpreter present at the time discharge medication and/ or follow-up instructions were provided. The mean comorbidity score for patients was 8.7 (SD 4.9). Sixty-six percent of patients reported having difficulty with at least one IADL posthospitalization. Forty-nine percent of patients reported having difficulty with three or more IADLs, and $35.1 \%$ had difficulty with three or more ADLs. Seventy-seven percent of patients were discharged to home, $19.6 \%$ to SNF/rehab/hospice, and $3.8 \%$ to another hospital (Table 2).

\section{Association of Caregiver and Patient Characteristics with Caregiver Perceived Stress Level}

In bivariate analysis, moderate/high caregiver perceived stress was significantly associated with older patients, more patient comorbidities, and patient preferred Chinese language (Tables 1 and 2). After backward stepwise elimination, our multivariate logistic regression model was adjusted for patient age, patient financial hardship, caregiver relationship to patient, caregiver MOS tangible support score, patient preferred language, hospital discharge floor, and discharge destination. Caregivers had greater odds of self-reporting moderate/high stress when caring for patients who preferred to receive medical care in Chinese rather than in English (OR 9.52; $p=$ 0.033 ) or when caring for patients discharged to another hospital rather than those caring for patients discharged to home or SNF/rehab/hospice (OR 9.95; $p=0.025$ ). No other characteristics were significantly associated with moderate/ high self-reported stress in the multivariate model.

\section{DISCUSSION}

We found that a majority of informal caregivers for Chineseand Spanish-speaking patients with LEP recently discharged from the hospital are adult children or grandchildren, who take on multiple roles caring for their family member, both in the health care environment and at home. A number of caregivers, who themselves had LEP, assumed a direct role coordinating their relative's care and communicating on their behalf. 
Table 1 Characteristics of Caregivers for Patients with Limited English Proficiency Recently Discharged from the Hospital According to Caregiver Perceived Stress $(n=158)$

\begin{tabular}{|c|c|c|c|c|}
\hline & Total $(n=158)$ & $\begin{array}{l}\text { Low caregiver } \\
\text { stress }(n=95)\end{array}$ & $\begin{array}{l}\text { Moderate-to-high caregiver } \\
\text { stress }(n=63)\end{array}$ & $p$ \\
\hline \multicolumn{5}{|l|}{ Caregiver Characteristics } \\
\hline \multicolumn{4}{|l|}{ Relationship to patient } & 0.422 \\
\hline Spouse/partner & 33 & $20(60.6)$ & $13(39.4)$ & \\
\hline Adult child/grandchild & 109 & $63(57.8)$ & $46(42.2)$ & \\
\hline Other family/friend & 16 & $12(75.0)$ & $4(25.0)$ & \\
\hline Caregiver lives with patient & 93 & $55(59.1)$ & $38(40.9)$ & 0.883 \\
\hline Age (years), mean $\pm \mathrm{SD}$ & $47.1 \pm 14.7$ & $45.7 \pm 15.2$ & $49.3 \pm 13.6$ & 0.136 \\
\hline Female & 103 & $60(58.3)$ & $43(41.7)$ & 0.510 \\
\hline \multicolumn{5}{|l|}{ Place of birth } \\
\hline USA & 30 & $22(73.3)$ & $8(26.7)$ & \\
\hline Latin America & 38 & $29(76.3)$ & $9(23.7)$ & \\
\hline Asia & 89 & $43(48.3)$ & $46(51.7)$ & \\
\hline \multicolumn{4}{|l|}{ Preferred language to receive medical care } & 0.063 \\
\hline English & 92 & $59(64.1)$ & $33(35.9)$ & \\
\hline Spanish & 22 & $16(72.7)$ & $6(27.3)$ & \\
\hline Chinese & 44 & $20(45.5)$ & $24(54.5)$ & \\
\hline \multicolumn{4}{|l|}{ English-speaking ability } & 0.131 \\
\hline Not at all & 17 & $9(52.9)$ & $8(47.1)$ & \\
\hline Not well & 37 & $23(62.2)$ & $14(37.8)$ & \\
\hline Well & 49 & $24(49.0)$ & $25(51.0)$ & \\
\hline Very well & 55 & $39(70.9)$ & $16(29.1)$ & \\
\hline \multicolumn{4}{|l|}{ Highest level of education } & 0.977 \\
\hline Some high school or less & 37 & $22(59.5)$ & $15(40.5)$ & \\
\hline High school diploma & 29 & $18(62.1)$ & $11(37.9)$ & \\
\hline Some college or more & 89 & $54(60.7)$ & $35(39.3)$ & \\
\hline \multicolumn{4}{|l|}{ Health literacy } & 0.318 \\
\hline Adequate & 108 & $67(62.0)$ & $41(38.0)$ & \\
\hline Inadequate & 45 & $24(53.3)$ & $21(46.7)$ & \\
\hline \multicolumn{4}{|l|}{ Self-rated health } & 0.204 \\
\hline Excellent & 18 & $11(61.1)$ & $7(38.9)$ & \\
\hline Very good & 34 & $26(76.5)$ & $8(23.5)$ & \\
\hline Good & 57 & $34(59.6)$ & $23(40.4)$ & \\
\hline Fair & 42 & $23(54.8)$ & $19(45.2)$ & \\
\hline Poor & 5 & $1(20.0)$ & $4(80.0)$ & \\
\hline Very poor & 1 & $0(0.0)$ & $1(100.0)$ & \\
\hline \multicolumn{5}{|c|}{ Global financial strain: in the past 12 months, was there a time when you did not have enough money to pay your monthly bills? } \\
\hline Yes & 40 & $23(57.5)$ & $17(42.5)$ & 0.549 \\
\hline MOS score: tangible support (mean, SD) & $4.3 \pm 0.9$ & $4.4 \pm 0.8$ & $4.2 \pm 1.1$ & 0.345 \\
\hline MOS score: emotional support (mean, SD) & $4.0 \pm 1.2$ & $4.1 \pm 1.1$ & $4.0 \pm 1.2$ & 0.181 \\
\hline
\end{tabular}

Percentages are based on non-missing values. Caregiver's response to the question: "In the past week, how often have you felt stressed caring for patient?" was dichotomized as low stress: "none of the time" and "a little of the time"; moderate/high stress: "some of the time," "most of the time," and "all of the time." All percentages shown are calculated from row proportions

$A D L$, activities of daily living; IADL, instrumental ADL; MOS, Medical Outcomes Study (score, range 1-5); SCQ, Self-Administered Comorbidity Questionnaire (score, range 0-26)

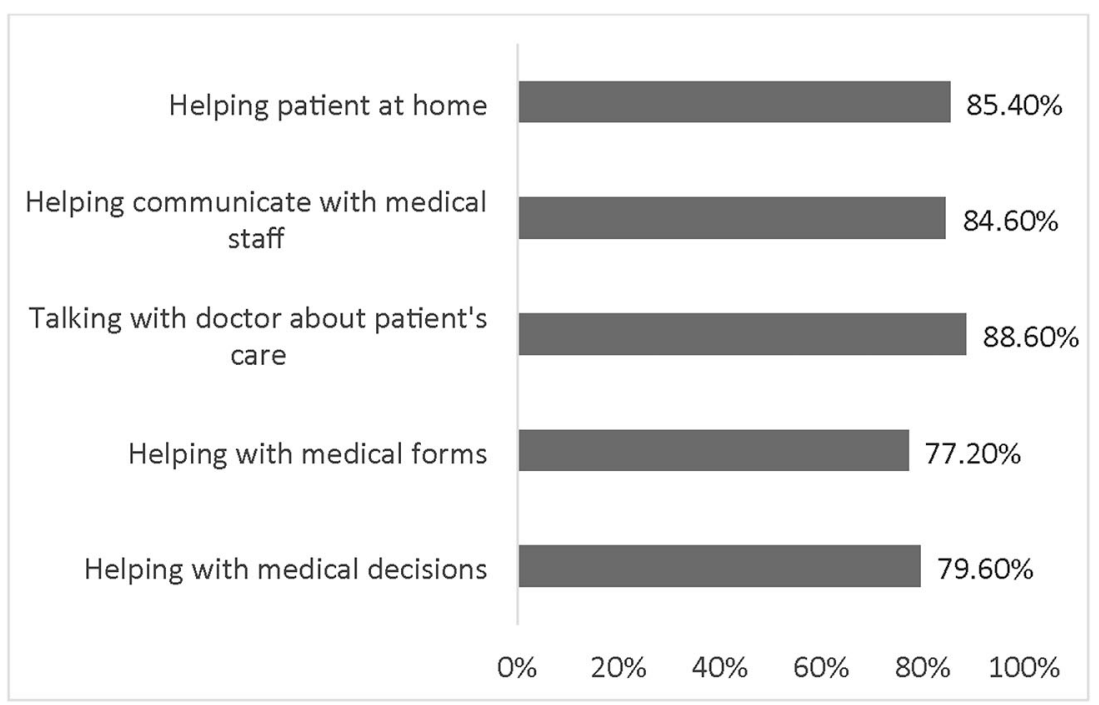

Figure 1 Caregiver-reported roles assisting patients with LEP post-hospitalization. 
Table 2 Characteristics of Patients with Limited English Proficiency Recently Discharged from the Hospital According to Caregiver Perceived Stress $(n=158)$

\begin{tabular}{|c|c|c|c|c|}
\hline & Total $(n=158)$ & $\begin{array}{l}\text { Low caregiver } \\
\text { stress }(n=95)\end{array}$ & $\begin{array}{l}\text { Moderate/high caregiver } \\
\text { stress }(n=63)\end{array}$ & $p$ \\
\hline \multicolumn{5}{|l|}{ Patient characteristics } \\
\hline Age (years), mean $\pm \mathrm{SD}$ & $69.6 \pm 13.2$ & $67.4 \pm 12.9$ & $73.0 \pm 12.9$ & 0.009 \\
\hline Female & 92 & $56(60.9)$ & $36(39.1)$ & 0.822 \\
\hline \multicolumn{4}{|l|}{ Preferred language to receive medical care } & \multirow[t]{4}{*}{0.008} \\
\hline English & 14 & $10(71.4)$ & $4(28.6)$ & \\
\hline Spanish & 37 & $30(81.1)$ & 7 (18.9) & \\
\hline Chinese & 107 & $55(51.4)$ & $52(48.6)$ & \\
\hline \multicolumn{4}{|l|}{ English-speaking ability } & \multirow[t]{5}{*}{0.074} \\
\hline Not at all & 78 & $41(52.6)$ & $37(47.4)$ & \\
\hline Not well & 65 & $46(70.8)$ & $19(29.2)$ & \\
\hline Well & 15 & $8(53.3)$ & $7(46.7)$ & \\
\hline Very well & 0 & 0 & 0 & \\
\hline Professional interpreter present at discharge & 19 & $13(68.4)$ & $6(31.6)$ & 0.431 \\
\hline \multicolumn{4}{|l|}{ Highest level of education } & \multirow{4}{*}{0.417} \\
\hline Some high school or less & 113 & $68(60.2)$ & $45(39.8)$ & \\
\hline High school diploma & 22 & $11(50.0)$ & $11(50.0)$ & \\
\hline Some college or more & 20 & $14(70.0)$ & $6(30.0)$ & \\
\hline \multicolumn{4}{|l|}{ Health literacy } & \multirow[t]{3}{*}{0.167} \\
\hline Adequate & 24 & $17(70.8)$ & $7(29.2)$ & \\
\hline Inadequate & 124 & $69(55.6)$ & $55(44.4)$ & \\
\hline SCQ comorbidity score (mean, SD) & $8.7 \pm 4.9$ & $8.0 \pm 4.7$ & $9.8 \pm 5.2$ & 0.029 \\
\hline \multicolumn{4}{|l|}{ Baseline IADL function } & \multirow[t]{4}{*}{0.070} \\
\hline No IADL impairments & 54 & $36(66.7)$ & $18(33.3)$ & \\
\hline Difficulty with $1-2$ IADLs & 26 & $19(73.1)$ & $7(26.9)$ & \\
\hline Difficulty with $\geq 3$ IADLs & 78 & $40(51.3)$ & $38(48.7)$ & \\
\hline \multicolumn{4}{|l|}{ Baseline ÅDL function } & \multirow[t]{4}{*}{0.276} \\
\hline No ADL impairments & 43 & $26(60.5)$ & $17(39.5)$ & \\
\hline Difficulty with 1-2 ADLs & 53 & $36(67.9)$ & $17(32.1)$ & \\
\hline Difficulty with $\geq 3$ ADLs & 52 & $23(44.2)$ & $29(55.8)$ & \\
\hline \multicolumn{4}{|l|}{ Hospital floor/service } & \multirow[t]{4}{*}{0.099} \\
\hline Cardiology & 89 & $47(52.8)$ & $42(47.2)$ & \\
\hline Orthopedics & 28 & $20(71.4)$ & $8(28.6)$ & \\
\hline General surgery & 41 & $28(68.3)$ & $13(31.7)$ & \\
\hline \multicolumn{4}{|l|}{ Hospital discharge destination } & \multirow[t]{4}{*}{0.393} \\
\hline Home & 121 & $74(61.2)$ & $47(38.8)$ & \\
\hline SNF/rehab/hospice & 31 & $19(61.3)$ & $12(38.7)$ & \\
\hline Another hospital & 6 & $2(33.3)$ & $4(66.7)$ & \\
\hline \multicolumn{5}{|c|}{ Global financial strain: in the past 12 months, was there a time when you did not have enough money to pay your monthly bills? } \\
\hline Yes & 52 & $33(63.5)$ & $19(36.5)$ & 0.549 \\
\hline
\end{tabular}

$A D L$, activities of daily living; IADL, instrumental ADL; MOS, Medical Outcomes Study (score, range 1-5); SCQ, Self-Administered Comorbidity Questionnaire (score, range 0-26)

Percentages are based on non-missing values. Caregiver's response to the question: "In the past week, how often have you felt stressed caring for patient?" was dichotomized as low stress: "none of the time" and "a little of the time"; moderate/high stress: "some of the time," "most of the time," and "all of the time." All percentages shown are calculated from row proportions

Among these caregivers, particularly those caring for Chinesespeaking patients and patients discharged to another hospital, we found moderate/high self-reported stress related to their caregiving role.

National surveys have provided a general description of informal caregivers. ${ }^{1-3}$ Similar to caregivers in the general population, in our sample, caregivers were mostly latemiddle-aged women caring for parents or grandparents. Almost all caregivers in our study had multiple roles caring for Chinese- and Spanish-speaking patients, including communicating with the health care team and assisting with medical decision-making. Notably, almost half of caregivers had a language barrier. For caregivers with LEP, navigating the complexities of the health care system alongside patients with LEP likely presents significant communication challenges.

Patients with LEP are at risk for adverse health events, longer hospital stays, and readmissions, compared with English-fluent patients. ${ }^{20,21}$ These disparities may be compounded for elderly patients, who often have complex health needs that require greater care coordination, particularly during the transition from hospital to home. ${ }^{22}$ Many caregivers in our study reported roles helping patients communicate with doctors and medical staff. We found that only $12 \%$ of caregivers with LEP had access to professional interpreters at the time discharge medication and follow-up instructions were provided. Although caregivers in our study had a relatively higher health literacy compared with the general population, many also had LEP. ${ }^{23}$ Caregivers facing language barriers may have difficulty helping patients overcome challenges communicating with their health care team, particularly when the teams do not access professional interpreters.

Asian caregivers have been found to have more care recipients than other racial/ethnic groups. ${ }^{10}$ Asian caregivers in the National Alliance for Caregiving (NAC) and American Association of Retired Persons (AARP) 
survey were also more likely to work full time while caregiving compared with White, Black, or Latino caregivers. ${ }^{1}$ In our study, we found that caregivers caring for Chinese-speaking patients were more likely to report moderate/high stress compared with those caring for English- and Spanish-speaking patients. More than half of caregivers we studied had at least some college education. A recent California Health Interview Survey analysis found that while having more education mitigates general psychological distress among White caregivers, it has been associated with more distress for Asian caregivers. ${ }^{24}$

While prior work has found an association between longer duration of caregiving and high caregiver stress, there has been a limited investigation of acute care scenarios. ${ }^{7}$ In our study, we focused on the experiences of caregivers in the acute post-hospital care setting. Post-hospital care is particularly important given the potential for patients having new, challenging functional limitations that increase caregiver burden. Among patients in our sample, nearly half had difficulty with 3 or more IADLs and one-third with 3 or more ADLs. Caregivers providing more assistance with ADLs are more likely in some cases to report overload in their caregiving role. ${ }^{25}$ Latino caregivers, however, have been found to report less stress related to caregiving than non-Latino caregivers despite providing more ADL assistance and more hours caregiving. ${ }^{26}$ In our bivariate analysis, we did not find a significant association of patient functional status with perceived caregiver stress levels. Given many of the patients in our study were discharged post-op, they likely had access to home health services or nursing at SNF/rehab/hospice, which may have alleviated caregiver perceived stress due to patients' functional limitations. Additionally, patients' functional limitations may in many cases have been temporary and improving during the post-procedure period.

Caregivers responsible for patients discharged to another hospital, as opposed to home, or SNF/rehab/hospice, reported moderate/high levels of perceived stress. This stress may have been due to these patients having more functional limitations or health complications, demanding a higher level of caregiver involvement upon discharge. In addition, given that caregivers were contacted 3 weeks after initial hospital discharge, these caregivers may have been interviewed soon after the patient's second hospital stay, further intensifying the perceived burden of care.

There are important limitations to consider when reviewing our findings. This is a small cross-sectional study, from which we cannot draw causal conclusions. Additionally, we relied on patients to identify one caregiver present at the time of discharge though these roles may have been dynamic or shared across caregivers. However, the vast majority of caregivers in our study reported performing three or more caregiver roles, suggesting that they were in fact the patient's primary caregiver. Also, by focusing on a distinct time period post-hospital discharge, we were able to limit the likelihood of multiple caregivers being closely involved in the patient's care. An additional limitation is that all caregiver roles assisting patients were self-reported. Yet, in terms of assessing our primary outcome, caregiver stress, self-described roles, and responsibilities may be the most relevant. We had a significant number of surrogates or proxies, most of whom also served as caregiver, respond on behalf of patients. This is not surprising given our study population was primarily post-op and prone to acute delirium. Proxies have been shown to overestimate health and functional limitations, particularly among elderly and disabled populations. ${ }^{27}$ Our interview questions, however, primarily focused on the caregiver's perceived quality of life and mood. We objectively measured patient comorbidities, demographic characteristics, admission, and discharge location. Only 6 patients in our study were discharged to another hospital so our finding that discharge to another hospital is associated with moderate/high caregiver perceived stress may not be clinically significant. For the assessment of caregiver perceived stress, we did not use a validated caregiver stress instrument in order to ease survey burden and because existing measures of caregiver burden have not been validated in populations with LEP. A national study of Latino family caregivers did, however, use a similar 1-question screen for caregiver stress as in our study. ${ }^{26}$ Also, we separately asked caregivers about emotional and tangible support as well as self-reported health questions included in longer, validated caregiver stress assessment tools.

Despite these limitations, this is the first study, to our knowledge, to describe characteristics and roles of caregivers for Chinese- and Spanish-speaking patients with LEP. Our study contributes to the limited literature examining the experiences of racially/ethnically diverse caregivers by providing insight into their linguistic diversity and its relationship to caregiver stress. By doing so, we can begin to develop effective tools and systems that will support all caregivers in their roles as part of the patient care team. Our results also suggested a missed opportunity to access professional interpreters at the time of hospital discharge to communicate with both patients and their caregivers with LEP, so as to avoid potential communication errors.

Contributors: Study concept and design: Karliner and Napoles; acquisition of subjects and/or data: Karliner; analysis and interpretation of data: Livaudais-Toman, Semere, Karliner, and Gregorich; and preparation of the manuscript: Semere, Karliner, Livaudais-Toman, Napoles, and Gregorich. No other individuals contributed substantially to this research or to the preparation of this manuscript.

Corresponding Author: Leah Karliner, MD, MAS; UCSF, 1545 Divisadero, San Francisco, CA 94143, USA (e-mail: leah. karliner@ucsf.edu).

Funders Dr. Nápoles' contribution was supported partially by the Division of Intramural Research, National Institute of Minority Health and Health Disparities; National Institute on Aging. This study was funded by a grant from the National Institute on Aging (R01AG038684), National Institute of Health. 


\section{Compliance with Ethical Standards:}

Conflict of Interest: The authors declare that they do not have a conflict of interest.

Disclaimer: The NIH had no role in the data collection, analysis, or writing of the manuscript. The contents and views in this manuscript are those of the authors and should not be construed to represent the views of the National Institutes of Health.

\section{REFERENCES}

1. Caregiving in the United States 2015. National Alliance for Caregiving and AARP (online). Available at: http://www.caregiving.org/caregiving2015/. Accessed March 25, 2019.

2. Adelman RD, Tmanova LL, Delgado $\mathbf{D}$ et al. Caregiver burden. A clinical review. JAMA. 2014;311(10):1052-1059.

3. Pinquart $\mathbf{M}$, Sörensen $\mathbf{S}$. Differences between caregivers and noncaregivers in psychological health and physical health: a metaanalysis. Psychol Aging. 2003;18(2):250-267.

4. Ho SC, Chan A, Woo $\mathbf{J}$ et al. Impact of caregiving on health and quality of life: a comparative population-based study of caregivers for elderly persons and noncaregivers. J Gerontol A Biol Sci Med Sci. 2009;64:873-879.

5. Principi A, Lamura G, Sirolla C et al. Work restrictions experienced by midlife family caregivers of older people. Ageing Soc. 2014;34(2):209-231.

6. Bauer JM, Sousa-Poza A. Impacts of informal caregiving on caregiver employment, health, and family. J Popul Ageing. 2015;8(3):113-145.

7. Bien-Barkowska K, Doroszkiewicz $\mathbf{H}$, Bien B. Silent strain of caregiving: exploring the best predictors of distress in family carers of geriatric patients. Clinical Interventions Aging. 2017;12:263-274.

8. Abdollahpour I, Nedjat $\mathbf{S}$, Noroozian $\mathbf{M}$ et al. Caregiver burden the strongest predictor of self-rated health in caregivers of patients with dementia. J Geriatr Psychiatry Neurol. 2014;27(3):172-180.

9. Nápoles AM, Chadiha L, Eversley R, Moreno-John G. Developing culturally sensitive dementia caregiver interventions: are we there yet? Am J Alzheimer's Dis Dementias., 2010;25(5):389-406.

10. Rote SM, Moot H. Racial/Ethnic Differences in Caregiving frequency: Does Immigrant Status Matter? J Gerontol B Psychol Sci Soc Sci. 2016; 00(00): 1-11.

11. Divi C, Koss RG, Schmaltz SP et al. Language proficiency and adverse events in US hospitals: A pilot study. Int $\mathrm{J}$ Qual Health Care. 2007;19(2):60.

12. Karliner LS, Pérez-Stable EJ, Gregorich SE. Convenient Access to Professional Interpreters in the Hospital Decreases Readmission Rates and Estimated Hospital Expenditures for Patients With Limited English Proficiency. Med Care. 2017;55(3):196-206.

13. Lee JS, Napoles A, Mutha S, Perez-Stable EJ, Gregorich S, LivaudaisToman J, Karliner LS. Hospital discharge preparedness for patients with limited English proficiency: A mixed methods study of bedside interpreter-phones. Patient Educ Couns. 2018; 101(1):25-32.

14. Karliner LS, Napoles-Springer AM, Schillinger D et al. Identification of limited English proficient patients in clinical care. J Gen Intern Med. 2008 23;(10): 1555-60.

15. Borson S, Scanian JM, Chen $\mathbf{P}$ et al. The Mini-Cog as a screen for dementia: validation in a population-based sample. J Am Geriatr Soc. 2003;51(10): 1451-4.

16. Sangha $\mathbf{O}$, Stucki G, Liang MH et al. The Self-Administered Comorbidity Questionnaire: a new method to assess comorbidity for clinical and health services research. Arthritis Rheum. 2003;49(2):156-63.

17. Chew LD, Griffin JM, Partin MR, et al. Validation of Screening Questions for Limited Health Literacy in a Large VA Outpatient Population. J Gen Intern Med. 2008;23(5):561-6.

18. Recommended SES Measures compiled by the Measurement and Methods Core of the Center for Aging in Diverse Communities. University of California, San Francisco, 2006 (online). Available at: https://accelerate.ucsf.edu/research/special-populations-measures. Accessed March 25, 2019.

19. Sherbourne CD, Stewart AL. The MOS social support survey. Soc Sci Med. 1991;32(6):705-14.

20. Karliner LS, Kim SE, Meltzer DO et al. Influence of language barriers on outcomes of hospital care for general medicine patients. J Hosp Med. 2010;5(5): 276-282.

21. John-Baptiste A, Naglie G, Tomlinson G et al. The effect of English language proficiency on length of stay and in-hospital mortality. J Gen Intern Med. 2004; 19:221-228.

22. Zurlo A \& Zuliani G. Management of care transition and hospital discharge. Aging Clin Exp Res. 2018; 30(3):263-270.

23. U.S. Department of Education, Institute of Education Sciences, 2003 National Assessment of Adult Literacy 2006 (online). Available at: https://nces.ed.gov/pubs2006/2006483.pdf. Accessed March 25, 2019.

24. Meyer OL, Liu X, Nguyen TN et al. Psychological Distress of Ethnically Diverse Adult Caregivers in the California Health Interview Survey. J Immigrant Minority Health. 2018: 20; 784.

25. Polenick CA, DePasquale N. Predictors of Secondary Role Strains Among Spousal Caregivers of Older Adults With Functional Disability. Gerontologist. 2018: 00 (00); 1-13.

26. Hispanic Family Caregiving in the U.S. Evercare and National Alliance for Caregiving 2008 (online). Available at: https://www.caregiving.org/data/ Hispanic Caregiver_Study_web_ENG_FINAL_11 04_08.pdf. Accessed March 25, 2019.

27. Li M, Harris I, Lu ZK. Differences in proxy-reported and patient-reported outcomes: assessing health and functional status among medicare beneficiaries. BMC Med Res Methodol. 2015;15:62.

Publisher's Note Springer Nature remains neutral with regard to jurisdictional claims in published maps and institutional affiliations. 